\title{
A Probabilistic-Based Portfolio Resampling Under the Mean-Variance Criterion
}

\author{
Anmar Al Wakil ${ }^{*}$ \\ *University of Paris-Est, France
}

Submitted: December 16, 2020 • Accepted: March 17, 2021

\begin{abstract}
An abundant amount of literature has documented the limitations of traditional unconstrained mean-variance optimization and Efficient Frontier (EF) considered as an estimation-error maximization that magnifies errors in parameter estimates. Originally introduced by Michaud (1998), empirical superiority of portfolio resampling supposedly lies in the addressing of parameter uncertainty by averaging forecasts that are based on a large number of bootstrap replications. Nevertheless, averaging over resampled portfolio weights in order to obtain the unique Resampled Efficient Frontier (REF, U.S. patent number 6,003,018) has been documented as a debated statistical procedure. Alternatively, we propose a probabilistic extension of the Michaud resampling that we introduce as the Probabilistic Resampled Efficient Frontier (PREF). The originality of this work lies in addressing the information loss in the REF by proposing a geometrical three-dimensional representation of the PREF in the mean-variance-probability space. Interestingly, this geometrical representation illustrates a confidence region around the naive EF associated to higher probabilities; in particular for simulated Global-Mean-Variance portfolios. Furthermore, the confidence region becomes wider with portfolio return, as is illustrated by the dispersion of simulated Maximum-Mean portfolios.
\end{abstract}

JEL classification: G11, C14, C15.

Keywords: Efficient Frontier, Mean-Variance Criterion, Portfolio Resampling, Bagging, Probabilistic Approach.

*Corresponding Author. E-mail: anmar.alwakil@gmail.com 


\section{Preliminary}

\subsection{Portfolio Selection under Uncertainty}

As are many economic decisions, portfolio selection problem is an uncertain decisionmaking process, in the sense of Knight (1921). Limitations of the traditional unconstrained mean-variance (MV hereafter) optimization are particularly well documented by an abundant amount of literature, including among others Michaud (1989), Frahm (2015), and Da Silva et al. (2009).

As stated by Markowitz (1952), classical MV optimization postulates that an investor has a preference for a portfolio of securities offering the maximum expected return for some given level of portfolio risk as measured by returns variance. MV Efficient Frontier (EF hereafter) defines the envelope curve containing the set of optimal portfolios for all possible levels of portfolio risk. Since it is assumed that inputs are without uncertainty and statistical estimation error; unconstrained MV optimizers are considered by Frahm (2015) as estimation-error maximizers magnifying errors associated to input estimates. Da Silva et al. (2009), among others, evidenced the instability of optimal solutions; since small changes in the inputs lead to large changes in the EF, in particular, when covariance matrix is ill-conditioned.

\subsection{Empirical Illustration}

We consider the 616 stocks from the MSCI U.S. Index and their market capitalizations as of June 2020. A set of 20 U.S. large cap stocks was randomly drawn from among the 100 largest stocks, with continuous monthly total returns over 20 years for the sample spanning from June 2000 to June 2020. Details of the dataset, including the description of the 20 stocks and their annualized returns and standard deviations, are displayed in Table 1. This standard methodology follows the literature, including - among others Michaud (1998), or Jobson and Korkie (1981).

Figure 1 illustrates the optimal portfolio allocation map that is related to the traditional unconstrained MV optimization; displaying optimal allocations from minimum risk on the left-hand side, to maximum risk on the right-hand side. Each color represents a particular stock from the selection universe. Furthermore, a vertical slice of the map corresponds to the MV optimal weights of each stock in the optimal portfolio at a specific level of portfolio risk. Naive MV optimal allocations exhibit sudden transitions from one risk to another, while 11 stocks among 20 in the selection universe are missing over the entire risk spectrum. From the investment perspective, naive MV portfolios are strongly concentrated, requiring ad hoc investment constraints to generate artificial portfolio diversification. This is particularly the case for the MM portfolio that is completely allocated in the stock ATVI.

Michaud (1989) documented some techniques that address the limitations of tradi- 
Figure 1: Optimal portfolio allocation map related to the classical unconstrained MV optimization

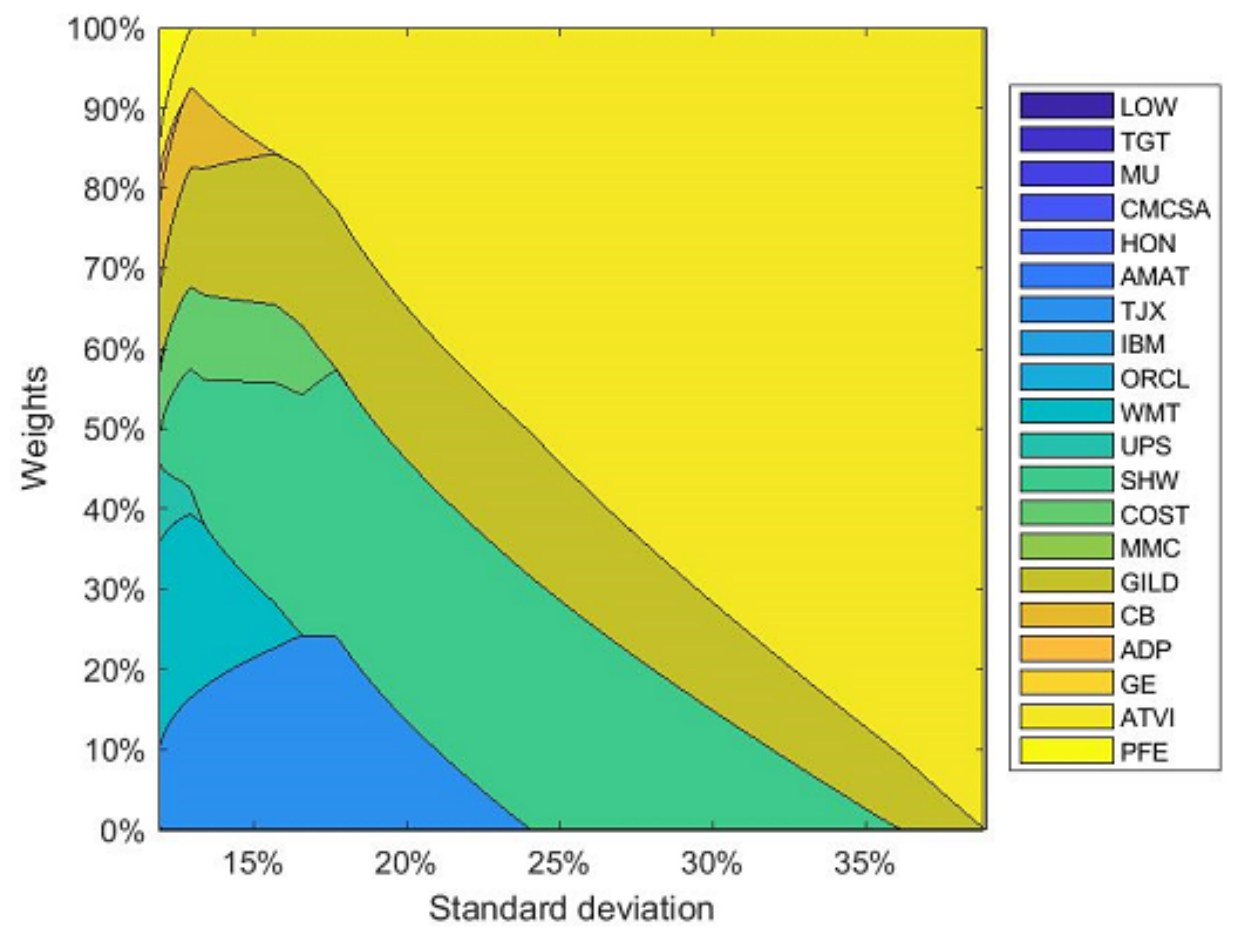

tional unconstrained MV optimization. Bayesian techniques assume the existence of a prior for estimation-error adjustment of inputs; whereas Michaud (1989) found the existence of a confidence region below the EF, where portfolios are statistically equivalent. Michaud (1998) invented (U.S. patent number 6,003,018) a portfolio resampling procedure that dominates the naive Markowitz approach, becoming one of the most debated findings in modern finance.

\section{Portfolio Resampling}

\subsection{General Setting}

In this section, we use some of the notations related to the portfolio-resampling procedure introduced by Michaud (1998) and Frahm (2015).

Theoretical superiority of resampled efficiency is assumed to be found by addressing the behavioral bias described by Mossin (1968); that investor decisions are particularly myopic in the naive Markowitz single-period optimization where he processes each time period as if it were the last one. Similarly, Frahm (2015) also found theoretical foundation of the Michaud procedure in the case in which the investor is considered a noise trader, whom prediction power is penalized by parameter uncertainty. Wolf (2007) relates the Michaud portfolio-resampling procedure to the bagging statistical technique developed by 
Breiman (1996). Consider any statistical method used to predict a variable when given some information on set $\aleph$; since $\aleph$ is stochastic, prediction suffers from estimation risk. Breiman (1996) proves that in the case of a quadratic loss function, estimation risk is lower when averaging the forecasts based on a large number of bootstrap replications of $\aleph$. In this sense, resampled efficiency lies in adding prediction variability, since bootstrap replications are drawn from an empirical, but not true, distribution of $\aleph$.

Definition 1. Let a sample $X=\left(X_{11}, \ldots, X_{m n}, \ldots, X_{M N}\right)$ of historical asset returns associated to $M$ observations and $N$ securities. Unknown parameters of true mean vector $\mu=\left(\mu_{1}, . ., \mu_{n}, \ldots, \mu_{N}\right)$ and covariance matrix $\Sigma=\left(\Sigma_{p q}\right)_{N \times N}$ are estimated by empirical moments of sample mean $\hat{\mu}=\left(\hat{\mu}_{1}, . ., \hat{\mu}_{n}, \ldots, \hat{\mu}_{N}\right)$, and covariance matrix $\widehat{\Sigma}=\left(\widehat{\Sigma}_{p q}\right)_{N \times N}$ is assumed to be symmetric and positive semidefinite. A sample $\widehat{X}=\left(\widehat{X}_{11}, \ldots, \widehat{X}_{m n}, \ldots, \widehat{X}_{M N}\right)$ of asset returns is generated by Monte Carlo from an $N$-dimensional multivariate normal distribution $N_{N}(\hat{\mu}, \widehat{\Sigma})$, where each component $\widehat{X}_{n}$ has distribution $N\left(\hat{\mu}_{n}, \hat{\sigma}_{n}^{2}\right)$ with variance $\hat{\sigma}_{n}^{2}=\widehat{\Sigma}_{n n}$. Parameter estimates $\hat{\mu}$ and $\widehat{\Sigma}$ are estimated by empirical moments $\hat{\hat{\mu}}$ and $\widehat{\widehat{\Sigma}}$, and optimal weights of I portfolios $\left(\hat{\hat{w}}_{1}, \hat{\hat{w}}_{2}, \ldots, \hat{\hat{w}}_{I}\right)$ on the EF are then calculated.

Definition 2. The first optimal portfolio $\hat{\hat{w}}_{1}$ is the Global Minimum-Variance (GMV hereafter) portfolio, i.e.

$$
\hat{\hat{w}}_{1}=\underset{w \in \Im}{\arg \min } w^{T} \widehat{\widehat{\Sigma}} w
$$

and the last optimal portfolio $\hat{\hat{w}}_{I}$ on the EF is the Maximum-Mean (MM) portfolio, i.e.

$$
\hat{\hat{w}}_{I}=\underset{w \in \Im}{\arg \max } w^{T} \hat{\hat{\mu}}
$$

The optimal portfolio $\hat{\hat{w}}_{s}$ for $s \in[1, I]$ is the Maximum Sharpe ratio (MS) portfolio, and for simplicity, risk-free rate is not considered, i.e.

$$
\hat{\hat{w}}_{s}=\underset{w \in \Im}{\arg \max } \frac{w^{T} \hat{\hat{\mu}}}{\sqrt{w^{T} \widehat{\hat{\Sigma}} w}}
$$

It is assumed that the set of feasible portfolios $\Im \subseteq\left\{w \in \mathbb{R}^{\mathbb{D}}: w^{T} \mathbf{1}=1, w \geq \mathbf{0}\right\}$ belongs to a $D$-dimensional Euclidean simplex. The other $I-2$ optimal portfolios maximize portfolio return $\hat{w}_{i}^{T} \hat{\hat{\mu}}$ such that $w_{i} \in \Im$ for some given level of standard deviation

$$
\sqrt{w_{i+1}^{T} \widehat{\widehat{\Sigma}} w_{i+1}}-\sqrt{w_{i}^{T} \widehat{\hat{\Sigma}} w_{i}}=\sqrt{w_{i}^{T} \widehat{\hat{\Sigma}} w_{i}}-\sqrt{w_{i-1}^{T} \widehat{\widehat{\Sigma}} w_{i-1}}
$$

$i=2, \ldots, I-1$

Definition 3. Let $\hat{\hat{w}}_{i j}$ the efficient portfolio $i=0,1, \ldots, I$ of draw $j=1, \ldots, J$, repeating the previous steps $J-1$ times and then averaging the I mean portfolios $\hat{\hat{w}}_{i j}$ of the $J$ simulated 
EF for each level of standard deviation $\hat{\hat{\sigma}}_{i}$ determines the efficient portfolios $\overline{\hat{w}}_{i}$ of the Resampled Efficient Frontier (REF hereafter); i.e.

$$
\overline{\hat{w}}=\frac{1}{J} \sum_{j=1}^{J} \hat{\hat{w}}_{i j},
$$

$i=0,1 \ldots, I$

For numerical purpose, the number of efficient portfolios considered on the EF is $I=100$ corresponding to the same grid of $I$ standard deviation points $\hat{\hat{\sigma}}_{i}$, and the number of draws considered for simulated EF is $J=1,000$.

Figure 2a illustrates the optimal portfolio allocation map related to the resampled MV optimization, displaying optimal allocations from minimum risk at the left-hand side, to maximum risk at the right-hand side. Resampled MV efficiency lies in some desirable properties when compared to naive MV efficiency. First, the optimal allocation map shows smoother transitions from one risk to another, including at significant levels all the 20 stocks from the selection universe. From the investment perspective, resampled MV portfolios that are more diversified than the naive MV portfolios that are displayed in Figure 1. This is exhibited by the MM portfolio that is allocated in five stocks; including ATVI (72\% vs 100\% in 1), SHW (13\%), TJX (7\%), MU (6\%), and GILD (2\%) as well as others.

Figure $2 \mathrm{~b}$ exhibits naive EF in the blue dotted curve and REF in the red dotted curve in the MV bidimensional space. This is from a minimum portfolio risk as seen on the left-hand side to maximum portfolio return as seen on the right-hand side. EF and REF are quite close in the MV space; specifically when considering the GMV portfolio. It suggests that optimizations produce quite similar optimal solutions. Portfolio resampling systematically both lowers and shortens the efficient frontier, lowering portfolio expected return and restricting portfolio risk to a narrower range.

\subsection{Theoretical Limitations}

Proclaiming that there is no theoretical argument as to why original portfolio resampling should dominate other portfolio optimization procedures; Scherer (2002) states "What is not clear, however, is why averaging over resampled portfolio weights should represent an optimal portfolio construction solution to deal with estimation error." Similarly, Markowitz and Usman (2003) dictate that the Michaud approach dominates the Bayesian strategies, but has "serious statistical and decision theoretic limitations."

For illustration; Figure 3 displays REF (red dotted curve), simulated EF (blue dotted curves), and GMV (yellow dot), MM (green dot), and MS (cyan dot) portfolios for each simulated EF. The REF curve is the single frontier that consists in the collection of the $I$ optimal portfolios $\overline{\hat{w}}_{i}$, calculated by averaging the $I$ mean portfolios $\left(\bar{w}_{1}, \bar{w}_{2}, \ldots, \bar{w}_{I}\right)$ 
Figure 2: Properties of portfolio resampling on the optimal allocation map and the representation in the mean-variance space

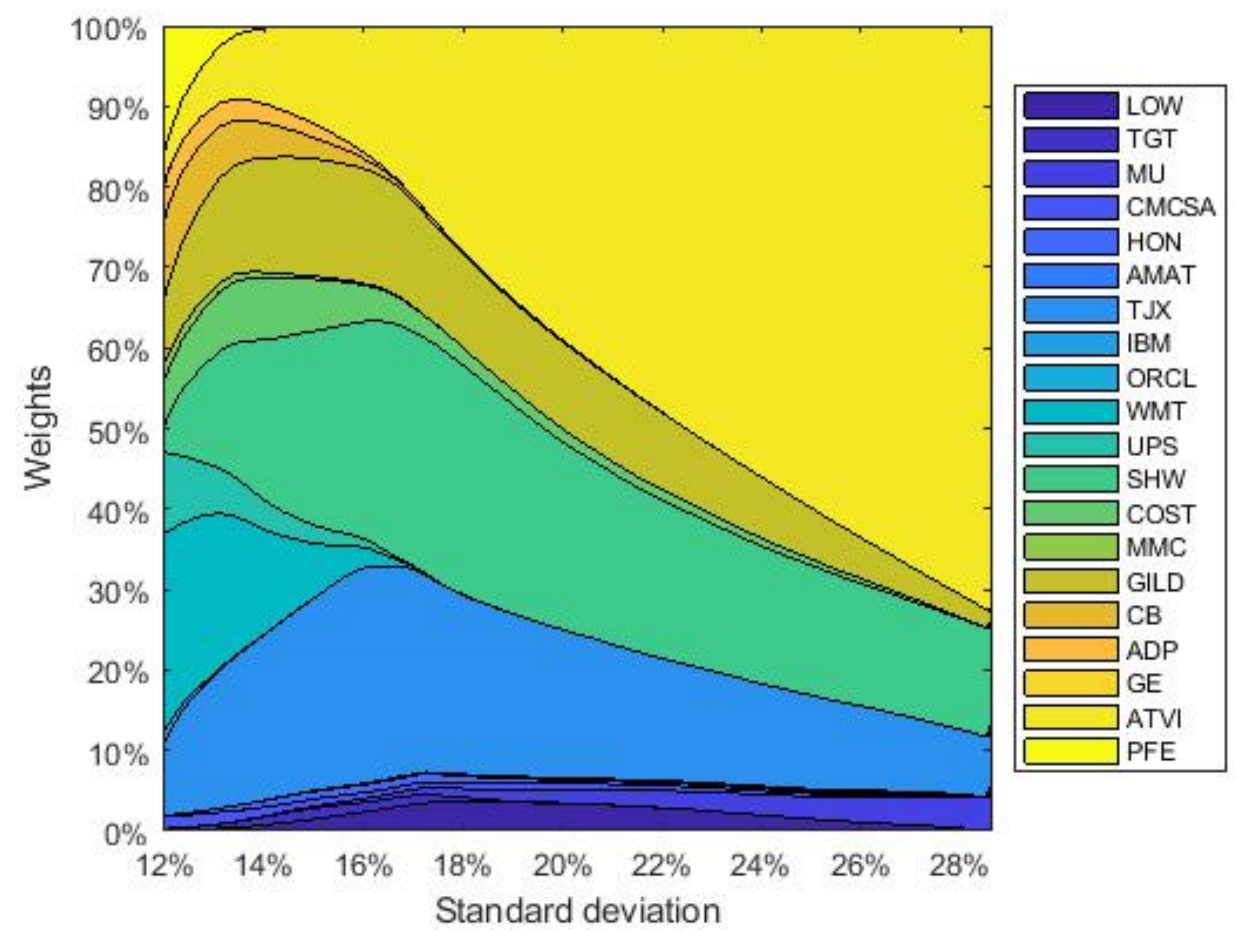

(a) Optimal portfolio allocation map related to the resampled MV optimization

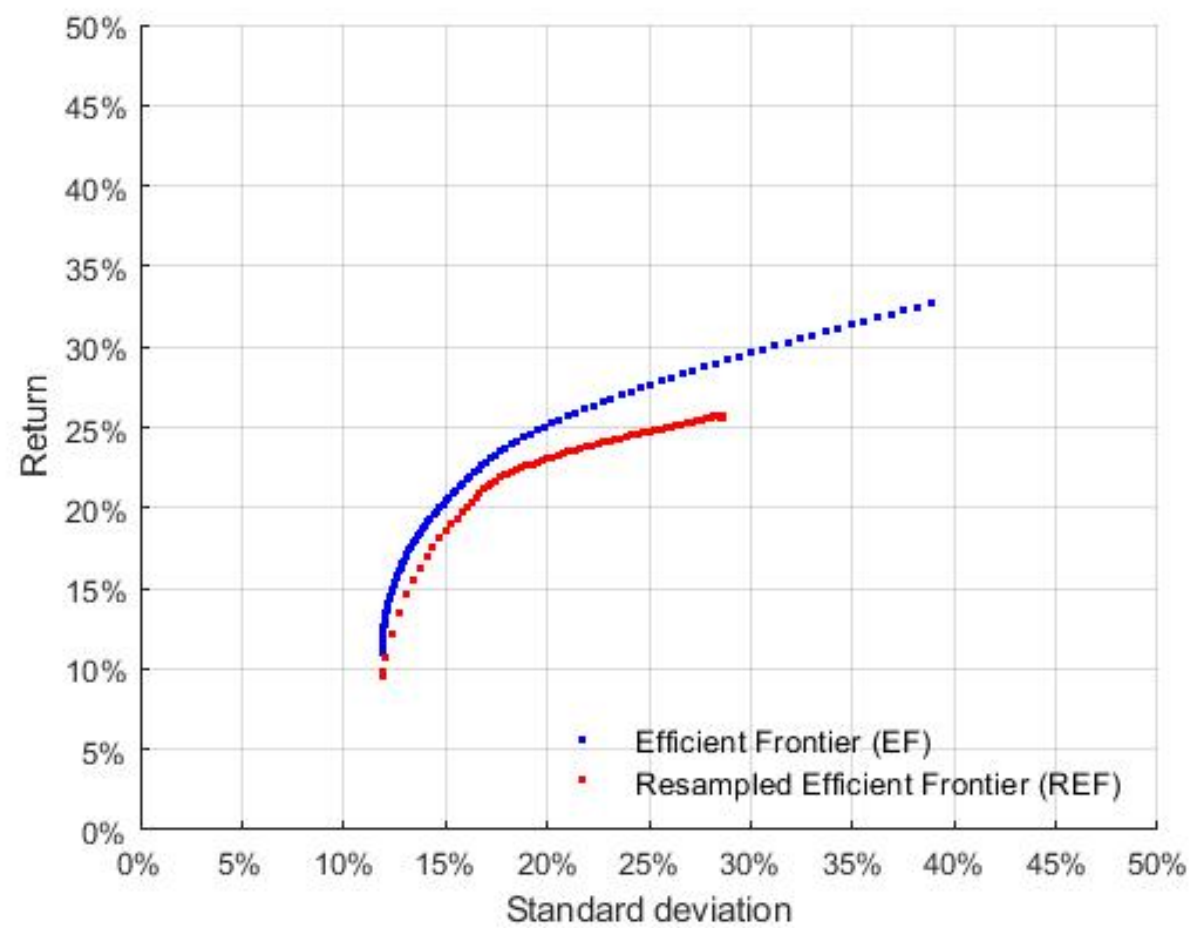

(b) Resampled Efficient Frontier and classical Efficient Frontier 
from the $J$ simulated EF curves. Special cases of optimal portfolios on the EF are GMV portfolios $\hat{\hat{w}}_{1}$, MM portfolios $\hat{\hat{w}}_{I}$, and MS portfolios $\hat{\hat{w}}_{s}$.

Figure 3: Resampled Efficient Frontier (REF), simulated Efficient Frontiers (EF), and special optimal portfolios

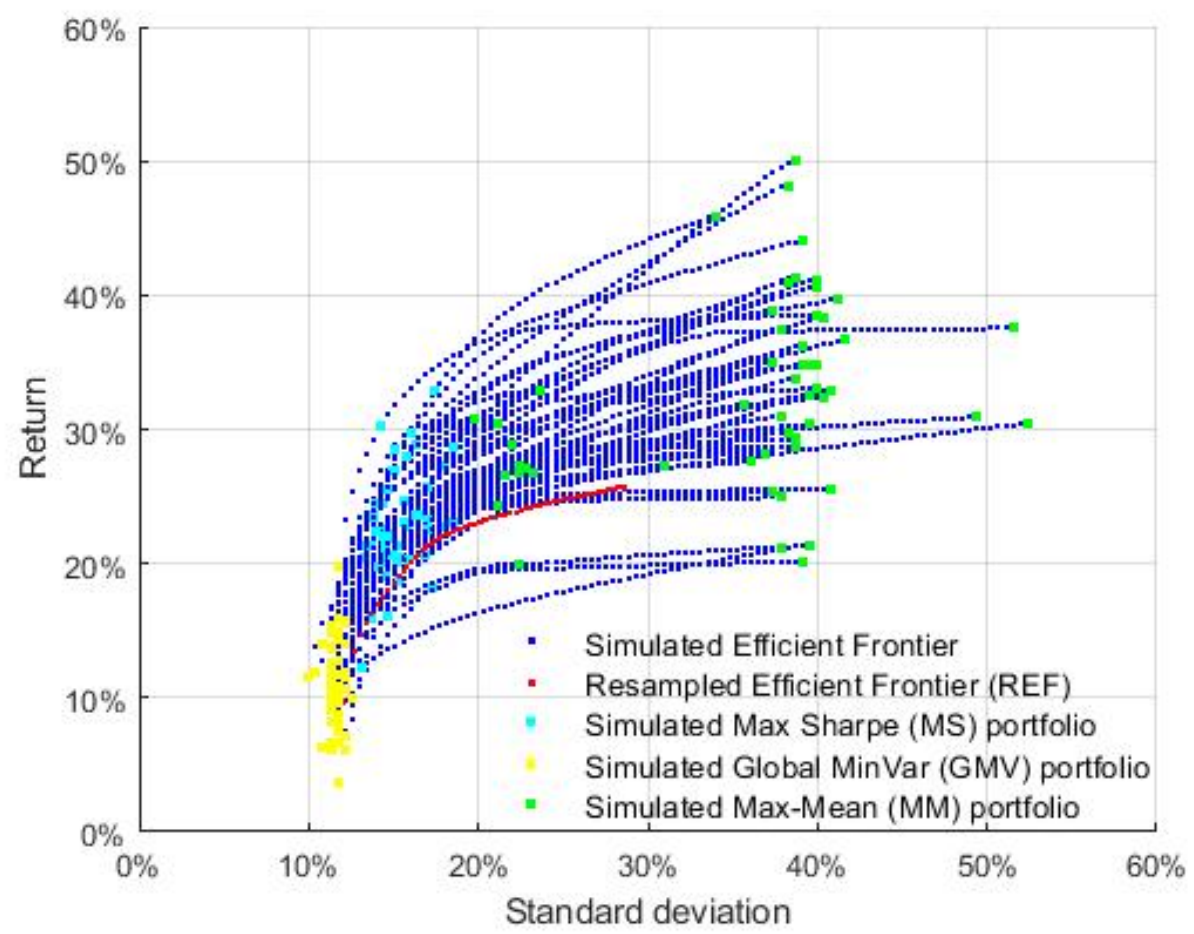

Figure 3 illustrates information loss when averaging over resampled weights; exhibiting a large region around the REF where assumed inefficient portfolios become statistically efficient at a specific level of probability. The confidence region grows as the portfolio expected return increases, reflecting a higher estimation error of the return and risk estimates. This is particularly evident when considering special cases of optimal portfolios, since MM (green dots) portfolios are substantially more dispersed across the meanvariance space than both MS (cyan dots) and consequently GMV (yellow dots) portfolios.

\subsection{Probabilistic Extension}

In this section, we propose a probabilistic extension for the Michaud resampling procedure. This is because averaging over resampled portfolio weights is documented as a debated statistical procedure in the literature. We use the notations related to the Kernel Density Estimator (KDE hereafter), which was introduced by Hill (1985).

Definition 4. Let the sample $\hat{\hat{M}}_{i}=\left(\hat{\hat{\mu}}_{i 1}, \hat{\hat{\mu}}_{i 2}, \ldots, \hat{\hat{\mu}}_{i J}\right)$ of $J$ empirical estimates of mean portfolio returns associated to efficient portfolios $\hat{\hat{w}}_{i j}=\left(\hat{\hat{w}}_{i 1}, \hat{\hat{w}}_{i 2}, \ldots, \hat{\hat{w}}_{i J}\right)$ for a same level 
of standard deviation $\hat{\hat{\sigma}}_{i}$. The true probability density functions $f_{\hat{\hat{M}}_{i}}=\left(f_{\hat{\bar{M}}_{1}}, f_{\hat{\bar{M}}_{2}}, \ldots, f_{\hat{M}_{I}}\right)$ of mean portfolio returns $\hat{\hat{M}}_{i}$ are estimated for $x \in \mathbb{R}$ by the kernel density estimators $\hat{f}_{\hat{\hat{M}}_{i}}=\left(\hat{f}_{\hat{\hat{M}}_{1}}, \hat{f}_{\hat{\bar{M}}_{2}}, \ldots, \hat{f}_{\hat{\hat{M}}_{I}}\right)$ defined as

$$
\hat{f}_{\hat{\hat{M}}_{i}}(x)=\frac{1}{n h} \sum_{j=1}^{J} K\left(\frac{x-\hat{\hat{M}}_{i}}{h}\right)
$$

where $K(\cdot)$ is the kernel smoothing function, $J$ is the sample size, and $h$ is the bandwidth parameter controlling the smoothness of the estimated probability density curve. Empirical estimate of probability mass function $\hat{p}_{i}(m)$ at standard deviation $\hat{\hat{\sigma}}_{i}$ is

$$
\hat{p}_{\hat{\hat{M}}_{i}}(m)=\mathbb{P}\left(\hat{\hat{M}}_{i}=m\right)=\int_{u \in \mathbb{R}} \hat{f}_{\hat{\hat{M}}_{i}}(u) d u
$$

Let $\Phi: \Im \rightarrow \mathbb{R}^{3}$ be the function mapping feasible efficient portfolios and defined by $\Phi\left(\hat{\hat{w}}_{i j}\right)=\left(\hat{\hat{M}}_{i}, \hat{\hat{\sigma}}_{i}, \hat{p}_{\hat{\hat{M}}_{i}}\right)$. Function $\Phi$ defines the Probabilistic Resampled Efficient Frontier (PREF hereafter), mapping the functions for empirical estimates of mean portfolio returns $\hat{\hat{M}}_{i}$, standard deviation $\hat{\hat{\sigma}}_{i}$, and probability $\hat{p}_{\hat{\hat{M}}_{i}}$ of efficient portfolios $\hat{\hat{w}}_{i j}$, i.e. MeanVariance-Probability (MVP) space.

Figure 4 illustrates the geometrical three-dimensional representation of the PREF in the space $\Phi\left(\hat{\hat{w}}_{i j}\right)=\left(\hat{\hat{M}}_{i}, \hat{\hat{\sigma}}_{i}, \hat{p}_{\hat{\hat{M}}_{i}}\right)$. Yellow areas are associated to higher estimated probabilities of realization of the mean-variance couple $\left(\hat{\hat{M}}_{i}, \hat{\hat{\sigma}}_{i}\right)$. The contour representation exhibits a confidence region around the naive EF of Figure1, which is associated to higher probabilities of realization. This observation is also illustrated in the three-dimensional surface by the yellow ridge region around the naive EF. Besides, probabilities are the highest around the simulated GMV portfolios $\hat{\hat{w}}_{1}$ as is clearly exhibited by the contour representation, and the confidence region becomes wider with expected portfolio return, as is illustrated by the substantial dispersion of simulated MM portfolios $\hat{\hat{w}}_{I}$ in Figure 3 .

\section{Conclusion}

In this contribution, we proposed an extension of the portfolio resampling procedure introduced by Michaud (1998). This paper is based on the theoretical superiority of resampled efficiency; which is assumed to lie in addressing the well-known behavioral bias that investor decisions are particularly myopic in the naive Markowitz single-period optimization where he processes each time period as if it were the last one. The original Michaud portfolio-resampling procedure has been related to the bagging statistical technique. This includes mitigation of parameter uncertainty and estimation risk by averaging the forecasts based on a large number of bootstrap replications. Nevertheless, 
Figure 4: Geometrical three-dimensional representation of the PREF in the space $\Phi\left(\hat{\hat{w}}_{i j}\right)=\left(\hat{\hat{M}}_{i}, \hat{\hat{\sigma}}_{i}, \hat{p}_{\hat{\hat{M}}_{i}}\right)$, where estimated empirical probability $\hat{p}_{\hat{\hat{M}}_{i}}$ is illustrated by color bars

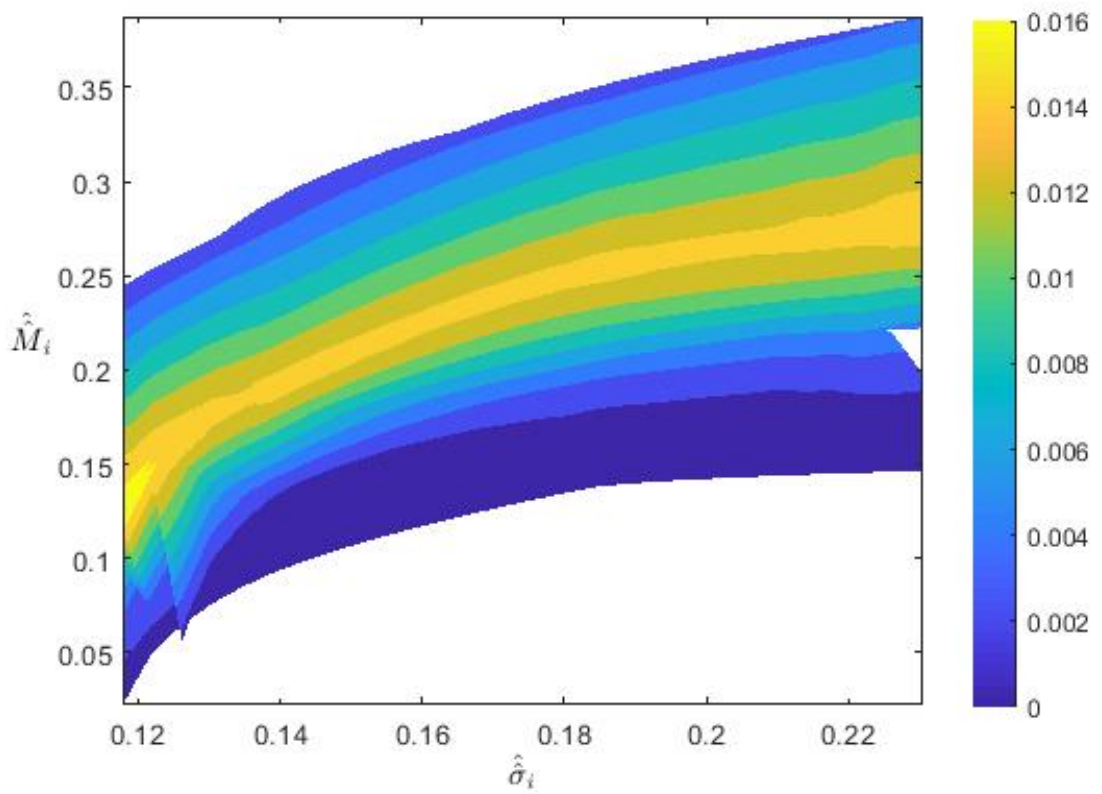

(a) Contour representation

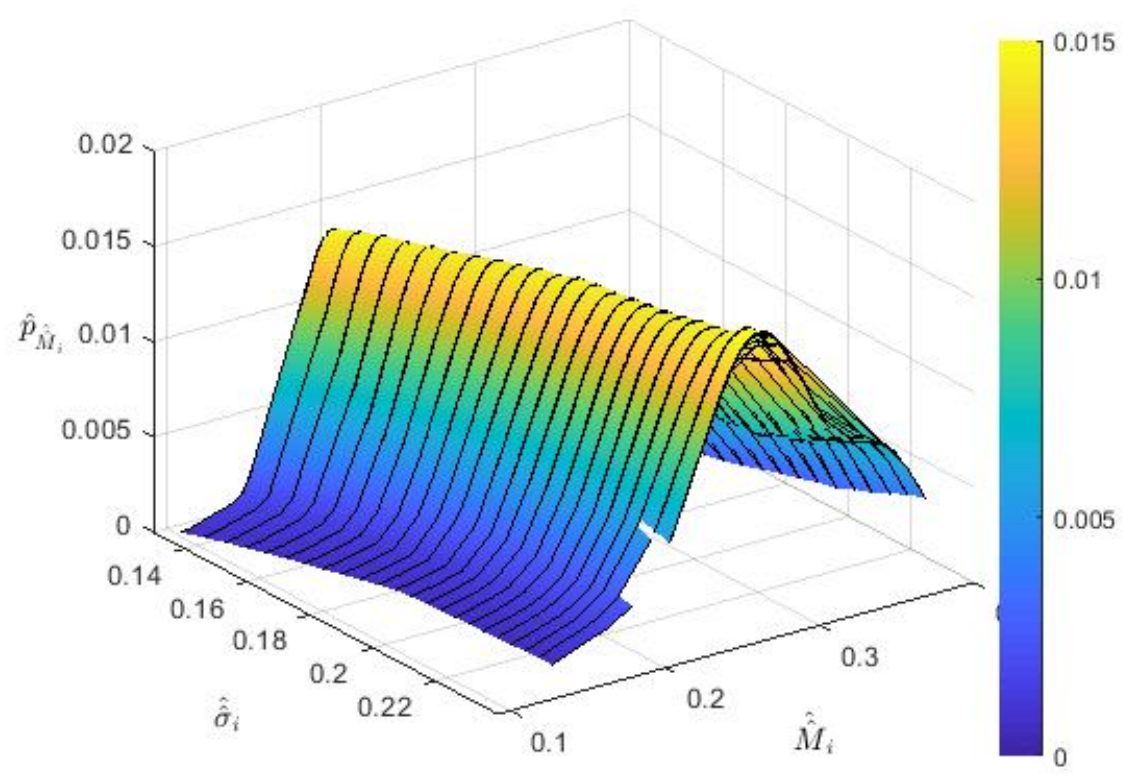

(b) Three-dimensional surface plot

there is an abundant amount of literature including Scherer (2002), or Markowitz and Usman (2003), as well as others, that have documented the statistical pitfalls of Michaud portfolio resampling, which was investigated as debated. There is no theoretical argu- 
ment why averaging over resampled portfolio weights in Michaud (1998) should dominate other portfolio optimization procedures. Information loss when averaging over resampled weights is illustrated by a large region around the Resampled Efficient Frontier (REF) of Michaud (1998); wherein assumed inefficient portfolios become statistically efficient at a specific level of probability.

This paper addresses the information loss of the REF when averaging over resampled portfolio weights by deriving a probabilistic extension of the REF that we introduce as the Probabilistic Resampled Efficient Frontier (PREF). The originality of this work lies in the geometrical three-dimensional representation of the PREF in the mean-varianceprobability (MVP) space, which is associated to the efficient portfolios. Interestingly, this geometrical representation illustrates a confidence region around the naive EF associated to higher probabilities of realization; specifically when considering the GMV portfolio. Furthermore, confidence region becomes wider with expected portfolio return, as is illustrated by the dispersion of MM portfolios. This theoretical framework appears useful to perform robust portfolio optimization by considering parameter uncertainty.

Following this work, we see further research in introducing a probabilistic approach for other portfolio techniques to exhibit confidence region around optimal portfolios.

\section{References}

Breiman, L. (1996). Bagging Predictors. Machine Learning, 24:123-140.

Da Silva, A. S., Lee, W., and Pornrojnangkool, B. (2009). The Black-Litterman Model for Active Portfolio Management. Journal of Portfolio Management, 35(2):61-70.

Efron, B. (2005). Bayesians, Frequentists, and Scientists. Journal of the American Statistical Association, 100(469):1-5.

Frahm, G. (2015). A Theoretical Foundation of Portfolio Resampling. Theory and Decision, 79(1):107-232.

Hill, P. (1985). Kernel Estimation of a Distribution Function. Communications in Statistics - Theory and Methods, 14(3):605-620.

Jobson, D. and Korkie, B. (1981). Putting Markowitz Theory to Work. Journal of Portfolio Management, 7(4):70-74.

Knight, F. H. (1921). Uncertainty and Profit. Hart, Schaffner and Marx.

Markowitz, H. (1952). Portfolio Selection. Journal of Finance, 7(1):77-91.

Markowitz, H. and Usman, N. (2003). Resampled Frontiers versus Diffuse Bayes. Journal of Investment Management, 1:1-17. 
Michaud, R. O. (1989). The Markowitz Optimization Enigma: Is 'Optimized' Optimal? Financial Analysts Journal, 45(1):31-42.

Michaud, R. O. (1998). Efficient Asset Management: A Practical Guide to Stock Portfolio Optimization and Asset Allocation. McGraw-Hill.

Michaud, R. O. and Michaud, R. (2003). An Examination of Resampled Portfolio Efficiency: A Comment and Response. Financial Analysts Journal, 59:15-16.

Michaud, R. O. and Michaud, R. (2008). Efficient Asset Management: A Practical Guide to Stock Portfolio Optimization and Asset-Allocation. Oxford University Press, New York, 2nd edition.

Mossin, J. (1968). Optimal Multiperiod Portfolio Policies. The Journal of Business, 41:215-229.

Politis, D. and White, H. (2004). Automatic Block-Length Selection for the Dependent Bootstrap. Econometric Reviews, 23(1):53-70.

Scherer, B. (2002). Portfolio Resampling: Review and Critique. Financial Analysts Journal, 58(6):98-109.

Wolf, M. (2007). Resampling vs. Shrinkage for Benchmarked Managers. Wilmott Magazine, 24:76-81. 


\section{Appendix}

Table 1: The dataset of 20 large cap stocks was drawn randomly from the 100 largest stocks in the MSCI U.S. Index and present 20 years of complete monthly total returns from June 2000 to June 2020. Returns and standard deviations are annualized

\begin{tabular}{llcc} 
Ticker & \multicolumn{1}{c}{ Company } & Return & Std deviation \\
\hline LOW & LOWE'S COS & 0.1811 & 0.2812 \\
TGT & TARGET CORP & 0.1242 & 0.2635 \\
MU & MICRON TECHNOLOGY & 0.1053 & 0.5013 \\
CMCSA & COMCAST CORP A (NEW) & 0.0961 & 0.2413 \\
HON & HONEYWELL INTERNATIONAL & 0.1365 & 0.2745 \\
AMAT & APPLIED MATERIALS & 0.0950 & 0.3648 \\
TJX & TJX COMPANIES & 0.1901 & 0.2202 \\
IBM & IBM CORP & 0.0573 & 0.2519 \\
ORCL & ORACLE CORP & 0.0685 & 0.3040 \\
WMT & WALMART & 0.0716 & 0.1810 \\
UPS & UNITED PARCEL SERVICE B & 0.0768 & 0.1974 \\
SHW & SHERWIN-WILLIAMS CO & 0.2078 & 0.2226 \\
COST & COSTCO WHOLESALE CORP & 0.1481 & 0.2041 \\
MMC & MARSH \& MCLENNAN COS & 0.0861 & 0.2187 \\
GILD & GILEAD SCIENCES & 0.2311 & 0.3097 \\
CB & CHUBB & 0.1205 & 0.2164 \\
ADP & AUTOMATIC DATA PROCESS & 0.1048 & 0.2011 \\
GE & GENERAL ELECTRIC CO & -0.0292 & 0.2867 \\
ATVI & ACTIVISION BLIZZARD & 0.3271 & 0.3892 \\
PFE & PFIZER & 0.0335 & 0.1914 \\
\hline
\end{tabular}

